\title{
Small-scale grassland assembly patterns differ above and below the soil surface
}

\author{
Jodi N. Price, ${ }^{1,3}$ Inga Hitesalu, ${ }^{1}$ Pille Gerhold, ${ }^{1,2}$ and Meelis Pärtel ${ }^{1}$ \\ ${ }^{1}$ Department of Botany, University of Tartu, Lai 40, 51005 Tartu, Estonia \\ ${ }^{2}$ Department of Experimental Plant Ecology, Institute for Water and Wetland Research, Radboud University Nijmegen, \\ P.O. Box 9010, 6500 GL Nijmegen, The Netherlands
}

\begin{abstract}
The existence of deterministic assembly rules for plant communities remains an important and unresolved topic in ecology. Most studies examining community assembly have sampled aboveground species diversity and composition. However, plants also coexist belowground, and many coexistence theories invoke belowground competition as an explanation for aboveground patterns. We used next-generation sequencing that enables the identification of roots and rhizomes from mixed-species samples to measure coexisting species at small scales in temperate grasslands. We used comparable data from above (conventional methods) and below (molecular techniques) the soil surface $(0.1 \times 0.1 \times 0.1 \mathrm{~m}$ volume). To detect evidence for nonrandom patterns in the direction of biotic or abiotic assembly processes, we used three assembly rules tests (richness variance, guild proportionality, and species co-occurrence indices) as well as pairwise association tests. We found support for biotic assembly rules aboveground, with lower variance in species richness than expected and more negative species associations. Belowground plant communities were structured more by abiotic processes, with greater variability in richness and guild proportionality than expected. Belowground assembly is largely driven by abiotic processes, with little evidence for competition-driven assembly, and this has implications for plant coexistence theories that are based on competition for soil resources.
\end{abstract}

Key words: belowground community assembly; guild proportionality; pairwise species interactions; pyrosequencing; root identification; species coexistence.

\section{INTRODUCTION}

Plant community assembly can be viewed as a process by which biotic and abiotic filters act on the regional species pool to determine the local community (Keddy 1992). A recent review found limited evidence for the existence of deterministic assembly rules, but the authors highlighted the need for methodological improvements (Götzenberger et al. 2012). To date, almost all studies on assembly rules have been conducted using aboveground data, mostly due to constraints in measuring belowground diversity. However, in many ecosystems, the majority of plant growth occurs belowground, and many coexistence theories highlight the importance of belowground competition for resources in driving aboveground patterns (Grime 1979, Tilman 1982). Moreover, only species that occur belowground can appear aboveground in any particular year, but not all species produce aboveground shoots every year. Hence, belowground communities are more stable and less affected by transient dynamics than aboveground communities; "real" species coexistence may be observed

Manuscript received 28 October 2011; revised 12 January 2012; accepted 23 January 2012. Corresponding Editor: J. Weiner.

${ }^{3}$ E-mail: Jodi.Price@ut.ee belowground, with the potential to provide greater insights into community assembly processes.

DNA-based techniques for identifying roots and rhizomes from root fragments and mixed-species samples have enabled the sampling of belowground diversity, and advanced our understanding of root ecology (Frank et al. 2010, Mommer et al. 2010, Kesanakurti et al. 2011, Hiiesalu et al. 2012). For rooted plants, aboveground diversity can only be a subset of belowground diversity. Indeed, Hiiesalu et al. (2012) found that belowground richness can be up to twice as high as aboveground richness, and with increasing sample size, aboveground richness reached an asymptote faster than belowground richness. Greater dispersion of roots and rhizomes in time and space can partially explain greater small-scale richness belowground (Wildová et al. 2007, Hiiesalu et al. 2012). In addition, Hiiesalu et al. (2012) found a nonlinear pattern between aboveground and belowground richness, suggesting aboveground community saturation. We suggest that belowground assembly can be the first step in the total community assembly process, and belowground communities can be viewed as a potential set of species from which the aboveground subset is filtered as a second step. Hence, belowground species are a small-scale analogy to the species pool in community ecology, where just a subset of the species pool actually occurs in a community, and the rest of the 
species form the dark diversity for the particular community (Pärtel et al. 2011).

One way in which we expect community assembly might differ above- and belowground relates to the relative symmetry of competitive interactions. Aboveground competition is usually size asymmetric, which means that larger plants can gain a disproportionate share of limiting resources (light), thereby increasing size differences and potentially resulting in competitive exclusion (Weiner 1990). Belowground competition has mostly been found to be size symmetric, with some exceptions (Fransen et al. 2001, Schenk 2006). Size asymmetric competition is more likely to result in competitive exclusion and hence should leave an "imprint" on spatial patterns. If competition is sizesymmetric belowground and competitive exclusion is reduced (as suggested by greater richness belowground; Hiiesalu et al. 2012), assembly patterns should also differ belowground, but to date no direct comparison of assembly patterns above- and belowground has been done.

Assembly rules are typically studied by inferring mechanisms through observed patterns, assuming that different processes will leave an imprint on spatial patterns. Randomization approaches are commonly used to study assembly rules; nonrandom patterns are interpreted as evidence for deterministic assembly processes, and random patterns are usually attributed to stochastic and dispersal-based assembly processes (for aboveground data, see a recent review by Götzenberger et al. [2012]). Various indices can be used to study assembly rules and most aim to infer the relative importance of biotic (mostly competition), and abiotic (e.g., environmental heterogeneity) processes in driving the observed patterns. To date, only two belowground community studies have used randomization approaches, both of which used species co-occurrence tests (Frank et al. 2010, Kesanakurti et al. 2011). We discuss three commonly applied assembly rules tests.

Classical niche theory predicts that the number of species in a community is limited by the number of niches (i.e., niche limitation; Wilson et al. 1987, Zobel and Zobel 1988). This is usually tested by examining the observed variance in species richness or diversity compared to a null model of random assembly (Wilson and Sykes 1988, Wilson and Whittaker 1995). Lower variance in species richness than expected by chance is generally interpreted as niche limitation because biotic interactions limit the co-occurrence of more species in a given habitat. Alternatively, higher variance than expected at random is usually attributed to abiotic assembly processes, such as environmental heterogeneity. A recent assembly rules review found lower variance in richness than expected in $39 \%$ of cases, in support of niche limitation, and greater variance than expected in $11 \%$ of cases (Götzenberger et al. 2012).

The concept of guild proportionality is based on the expectation that species are more similar within than between guilds. Hence, if competitive exclusion occurs, it will more likely occur within a guild, and the relative proportions of species in each guild should be relatively constant, i.e., less variance in guild proportionality than expected (Wilson 1989). A review based on aboveground data found support for guild proportionality in only $\sim 7 \%$ of cases (Götzenberger et al. 2012). An alternative hypothesis states that competitive exclusion acts on more dissimilar species and species bearing traits associated with low competitive ability may be excluded (Grime 2006, Schamp and Aarssen 2009), thereby increasing similarity among coexisting species.

Co-occurrence indices are used to detect nonrandom patterns in the direction of species segregation or aggregation. Recently, Götzenberger et al. (2012) found more support for species aggregation (29\% of cases) than segregation ( $12 \%$ of cases). Two studies have examined species co-occurrences in root communities at small scales, and contrasting results have been found (Frank et al. 2010, Kesanakurti et al. 2011). Frank et al. (2010) found that grassland species occurrences were randomly assorted at various soil depths, except at a relatively dry site where significant segregation was detected, largely driven by Festuca idahoensis. Kesanakurti et al. (2011) found strong species segregation in an old field, indicating that species are generally found in isolation. In this study, they used two different indices (c-scores and checker index) and found contrasting results in the top $20 \mathrm{~cm}$ of soil, with only the checker index showing significant segregation. These studies, however, did not directly compare above- and belowground patterns.

We applied three commonly used tests to examine evidence for assembly rules above- and belowground. Specifically, we examined species richness variance, guild proportionality, and species co-occurrences in a temperate mesic grassland in northern Europe, where belowground diversity patterns have already been described (Hiiesalu et al. 2012). In this study, mean species richness at small scales was high; for aboveground data this was 5.8 species $/ 0.01 \mathrm{~m}^{2}$ and 8.2 species $/ 0.01 \mathrm{~m}^{2}$ belowground to a depth of $0.1 \mathrm{~m}$ (Hiiesalu et al. 2012).

We hypothesize that patterns of plant assembly differ above- and belowground based on differences in the air and soil environment. We expect to find more evidence for biotic assembly rules aboveground, and hence we should find less variance in species richness than expected at random, more proportional guilds, and fewer co-occurrences between species. Belowground, due to the diverse nature of the soil environment and relatively symmetric resource competition, we expect to find more evidence of abiotic assembly processes such as environmental heterogeneity. Hence, we expect greater variability in species richness and guild proportionality, and more co-occurrences between species belowground.

\section{Methods}

We measured above- and belowground plant species richness in a 2-ha diverse mesophytic grassland in

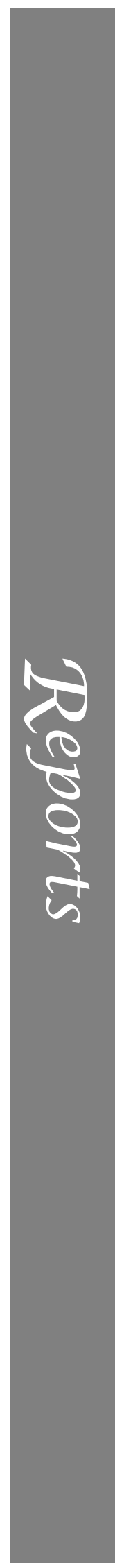


southeastern Estonia (Põlva County; $58^{\circ} 06^{\prime} \mathrm{N} ; 27^{\circ} 04^{\prime} \mathrm{E}$; see Plate 1). A detailed description of the study area is provided in Hiiesalu et al. (2012). Richness was recorded in 100 volumes $(0.1 \times 0.1 \times 0.1 \mathrm{~m})$ above and below the soil surface in mid-June 2007. Aboveground, this sampling unit corresponds with a $0.1 \times 0.1 \mathrm{~m}$ quadrat, since only species that were rooted in the quadrat were used in the data analysis. A total of 29 species were detected belowground and 22 aboveground (Hiiesalu et al. 2012). Quadrats were arranged contiguously in 10 randomly placed $1 \mathrm{~m}$ long transects, with 10 samples per transect. The spatial location of every quadrat was recorded. Aboveground species richness was determined by identifying all vascular plant species in each quadrat. Belowground species richness in each plot was measured by collecting a volume of soil $\left(0.001 \mathrm{~m}^{3}\right)$. The litter layer was removed and roots were sieved from the soil. Roots were crushed using liquid nitrogen, mixed well and a subsample was taken for DNA analysis. For belowground identification of species, 454 sequencing was used. The DNA region (chloroplast $\operatorname{trn} \mathrm{L}$ intron) used did not separate a few closely related species, and hence we sometimes used species groups. The same species were also merged in the aboveground data in order to have comparable taxonomic resolution. For more details of the molecular analysis see Appendix A or Hiiesalu et al. (2012).

\section{Data analysis}

All analyses were conducted on above- and belowground samples using presence-absence data. We defined the guilds as grasses (Poaceae) and forbs (all other families). Out of a total of 29 species, 6 species were grasses, but these species often had high frequency. All analyses were done in R ( R Development Core Team 2009).

We compared variance in richness, guild proportionality, and species co-occurrences (c-scores and checker index) in observed and randomized data sets (2000 randomizations). The checker index is based on the numbers of species pairs that never co-occur and c-scores are based on the degree of segregation in the data matrix (Gotelli 2000). Randomizations were spatially constrained, i.e., we randomized only within each transect of 10 samples. This prevents spurious effects from spatial autocorrelation (Legendre et al. 2004). As a rule, species were more abundant belowground than aboveground (Hiiesalu et al. 2012). Therefore we further examined aboveground community assembly by spatially constraining species' aboveground occurrences in randomizations to only those plots in which they actually occurred belowground. This enabled us to ask if any assembly rules acting aboveground restrict species appearance from the belowground community. For richness variance, we kept species frequencies constant, and for guild proportionality and species co-occurrences we kept both species richness and frequencies constant by applying the quasi- swap method of Miklos and Podani (2004), performed using the $\mathrm{R}$ package vegan (available online). ${ }^{4}$ Significance of deviations between the observed and randomized data sets were defined using the Monte Carlo method (by proportion of randomization when the parameter in the randomized data set was more extreme than in the observed data set).

We compared species' pairwise associations, taking into account spatial configuration using generalized estimating equations (GEE) for binary data. We treated the study site as a grid with a grain size equal to our plot size $\left(0.01 \mathrm{~m}^{2}\right)$ and determined if the presence of a species is positively or negatively associated with the presence of another species. For full details of the method and the $\mathrm{R}$ script used, see Carl and Kühn (2007). This test gave identical results independent of which species was used as an independent or dependent factor, and it effectively omitted spurious associations due to spatial autocorrelation. We also conducted a Fisher exact test to determine if there was a nonrandom pattern in the number of positive or negative interactions in our guilds, i.e., grasses-grasses, grasses-forbs, forbs-forbs.

\section{Results}

\section{Richness variance and guild proportionality}

Aboveground species richness was significantly less variable than expected at random (observed $<$ random, $P=0.01$ ), and when species were spatially constrained by their presence belowground these results were even more significant $(P<0.0001)$. In contrast, belowground data showed a tendency toward greater variance in richness than expected (observed $>$ random, $P=0.09$ ). We found no evidence for guild proportionality (grasses and forbs) aboveground (observed = random), but when species where spatially constrained by their belowground presence, guild proportionality was significant (observed $<$ random, $P<0.03$ ). In contrast, belowground we found significantly greater variance in guild proportionality than expected at random (observed $>$ random, $P=0.03)$.

\section{Species co-occurrences}

Aboveground species were significantly segregated based on c-scores (observed $>$ random, $P<0.001$ ), and this was more significant when species were constrained by their belowground presence $(P<0.0001)$. However, this was not significant based on the checker index, except when species were constrained by their belowground presence (observed $>$ random, $P=0.008$ ). Belowground plant species associations did not differ from random expectations based on both indices (observed $=$ random). Pairwise comparisons based on the presence and absence of all species pairs revealed many positive and negative species associations (i.e., aggregation and segregation, respectively) above- and

\footnotetext{
${ }^{4}$ http://CRAN.R-project.org/package=vegan
} 


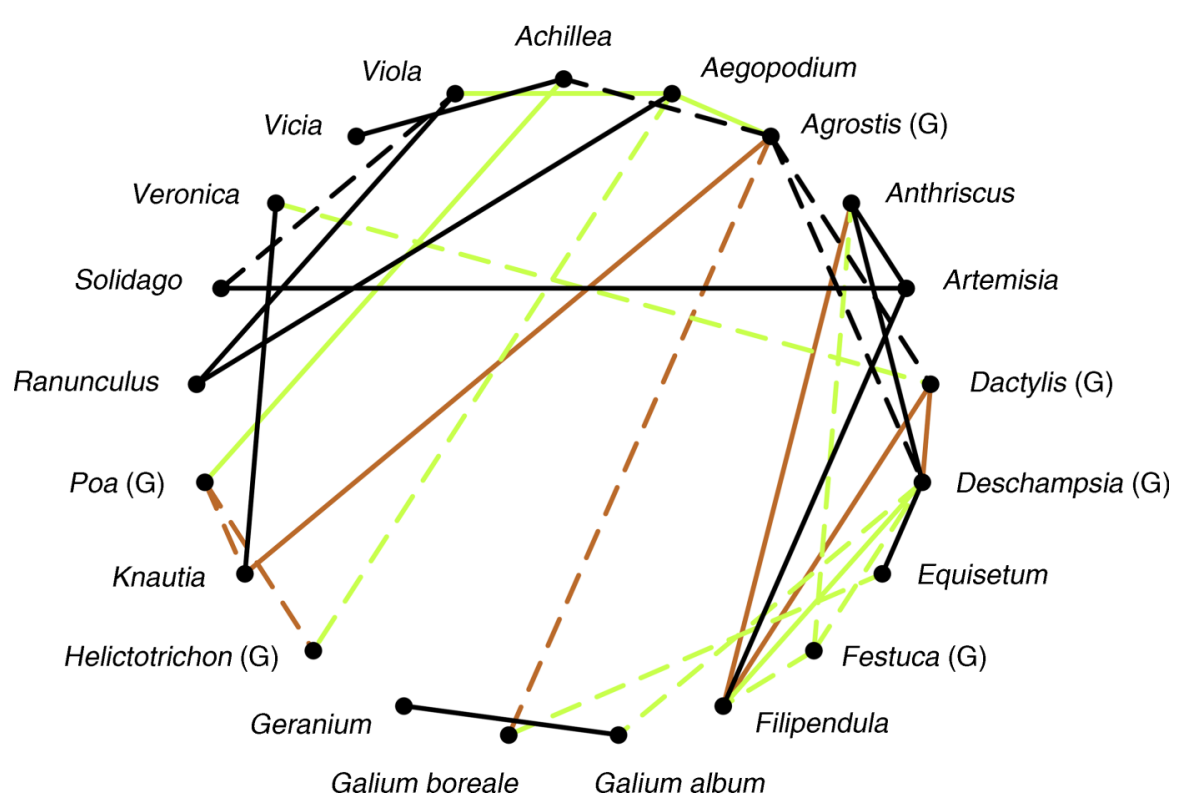

FIG. 1. Significant pairwise small-scale grassland species associations detected aboveground (green), belowground (black), or both above and belowground (brown) by spatially informed generalized estimation equations. Positive associations are indicated by solid lines, and negative associations by dashed lines. Full species names and $P$ values are in Appendix B. Grass species are denoted by $(\mathrm{G})$. Only species with significant interactions are shown.

belowground (Fig. 1, Appendix B). We found more species aggregation (14 species pairs) belowground than segregation (7 species pairs), whereas aboveground similar numbers of species pairs were significantly segregated and aggregated (10 and 8 species pairs, respectively). However, the c-scores suggest that the aboveground community is characterized by segregation, so these species pairs must be frequent enough to drive this pattern. We defined six types of species associations, depending on if associations were aggregated or segregated and if the association was found aboveground, belowground, or both (see Appendix B). We found the numbers of positive and negative associations (both above- and belowground) differed between three groups (grasses and grasses, grasses and forbs, forbs and forbs) (Fisher exact test, $P=0.037$ ). This was because most grass-grass interactions were negative (four negative, one positive), and most forbforb associations were positive (10 positive, two negative). We found equal numbers of positive and negative associations between grasses and forbs.

\section{Discussion}

Patterns in small-scale plant community assembly differed above and below the soil surface in a diverse mesophytic grassland. Aboveground, as predicted, we found more support for biotic assembly processes, as demonstrated by lower variance in species richness than expected at random, and species segregation, consistent with other aboveground studies (Gotelli and McCabe 2002, Götzenberger et al. 2012). We also found support for guild proportionality, but only when species were constrained by their belowground presence. Aboveground assembly appears to be driven mainly by biotic processes, presumably asymmetric light competition, and patterns were stronger in this direction when we constrained the tests by species belowground presence. Hence, biotic filters operate strongly to determine species presence aboveground.

Belowground, we found more support for assembly governed by abiotic and stochastic processes, as demonstrated by greater variance in richness and guild proportionality than expected and random species association patterns. The soil environment is more variable than the air environment, including gradients of different macro- and micro-nutrients, and chemical and physical conditions (e.g., $\mathrm{pH}$, soil particle size). This diversity of resources produces large variability in micro-environmental conditions, thereby promoting belowground coexistence, compared to aboveground resources, where mostly light conditions vary. Our results, and those of Hiiesalu et al. (2012), suggest that increased species coexistence belowground is partly because competitive exclusion is not occurring at the same spatial or temporal scale that it occurs aboveground. This is most likely due to the nature of soil environment and resource utilization compared to the air environment. Aboveground, the limiting resource (i.e., light) is unidirectional and non-storable, whereas, belowground resources can be acquired in all directions and are storable, and this probably enabled richness to increase at higher levels of productivity, even when aboveground richness declined in this grassland (Hiiesalu et al. 2012). This pattern may also be explained by 


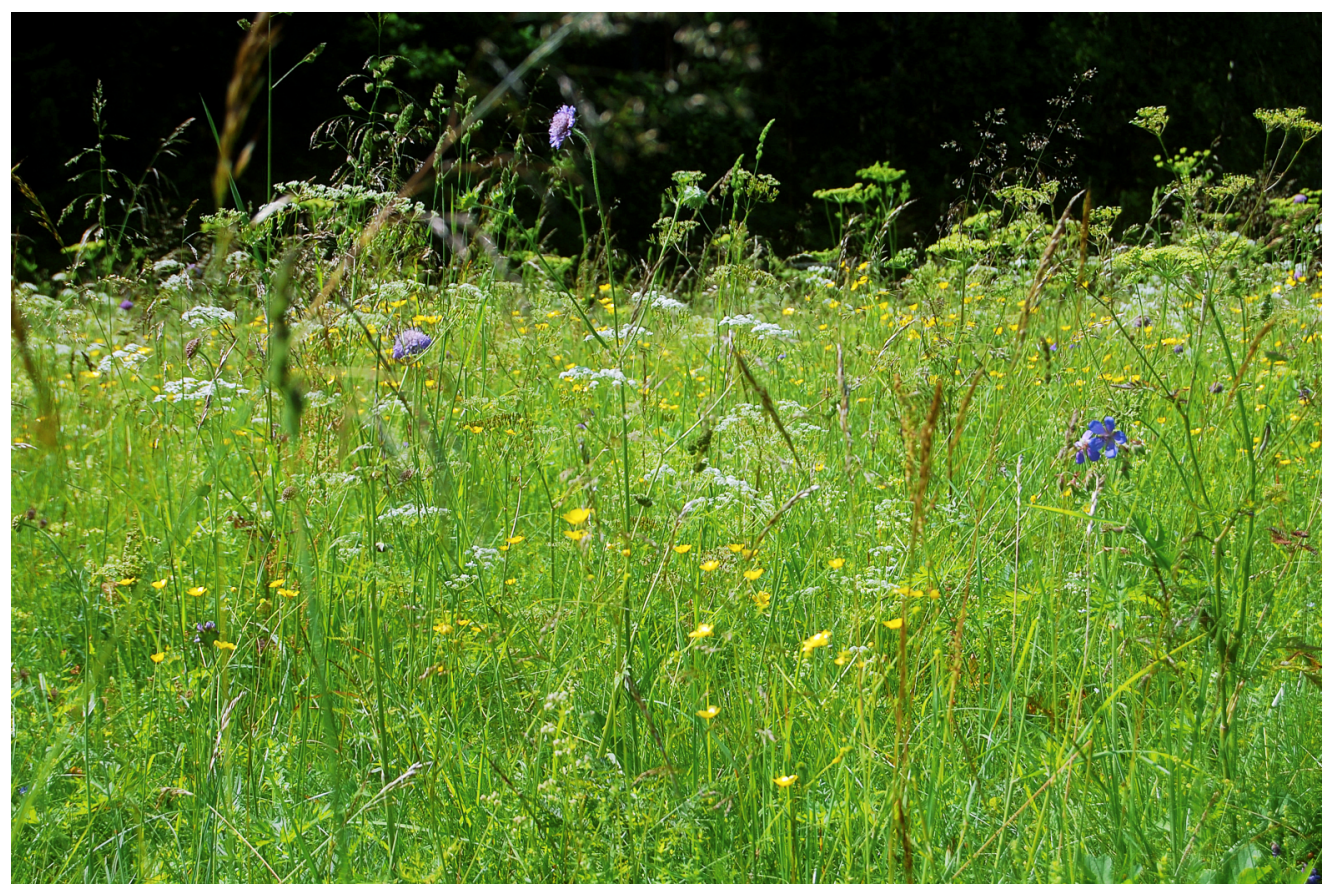

Plate 1. Species-rich mesophytic grassland in southeastern Estonia. Aboveground plant community assembly is largely driven by competition for light, whereas belowground communities were structured more by abiotic processes. Photo credit: Riin Tamme.

root foraging ability, as roots can preferentially grow into fertile patches, and the responses seem to be species specific (Farley and Fitter 1999, Hodge 2004, Mommer et al. 2012).

Greater variability in richness and guild proportionality belowground can also be due to biotic heterogeneity. For example, soil biota (i.e., microbes and fungi) create heterogeneous micro-patches in the soil (Hodge 2004, Maron et al. 2011, Schnitzer et al. 2011), which can influence vegetation either through competition for resources (Reynolds et al. 2003), or through nonresource-based biotic interactions, e.g., negative plantsoil feedbacks (Bever 2003, Petermann et al. 2010). The imprint that these interactions leave on plant community structure is largely unknown, but might not fit nicely into a division between abiotic and biotic processes as is commonly assigned using these assembly rules tests. Indeed, assigning mechanistic explanations to the patterns observed using these commonly applied assembly rules test is complicated because various processes can produce the observed patterns (Bell 2005, Seabloom et al. 2005, Götzenberger et al. 2012). For aboveground data, our results seem fairly robust and consistent with a large number of other studies finding evidence for niche limitation, and asymmetric light competition (Götzenberger et al. 2012). Root communities are shaped by many biotic interactions, in addition to resource competition, including facilitation, plant behavioral ecology, allelopathy, and interactions with other soil organisms (e.g., Callaway 1995, de Kroon 2007,
Semchenko et al. 2007a, $b$, Bever et al. 2010). Nonetheless, as aboveground assembly can only modify patterns from the belowground "species pool," examining spatial patterns in root communities should be a first step in detecting evidence of nonrandom processes governing community assembly.

Biotic assembly processes were also demonstrated aboveground by species co-occurrences, with more species segregation in c-scores and pairwise associations' tests. The negative species associations were driven by a few species that were abundant in the grassland community. In contrast, species segregation played a minor role in belowground assembly, with the exception of several species pairs that were negatively associated, consistent with Frank et al. (2010). In some cases, we found consistent pairwise associations both above- and belowground, suggesting that this pattern is driven by belowground interactions, and that aboveground assembly can be either neutral or driven by the same processes. Four species pairs had positive associations aboveground and no relationship belowground; the mechanism can be either aboveground facilitation or an overlap in microsite preferences. Seven species pairs were negatively associated aboveground, but no relationship was found belowground and this is likely due to competition for light. These two types of interactions are clearly driven by aboveground processes, and, in this case, we suggest that aboveground assembly modifies random patterns belowground. 
Belowground, we found 10 species pairs that were positively associated and had no associations' aboveground, hence belowground processes were driving the observed patterns. We offer several explanations for this pattern. First, positive associations belowground can be due to facilitation. For example, roots can increase the availability of resources for other species (Callaway 1995, Hauggaard-Nielson and Jensen 2005). Positive associations can also be due to root behavioral ecology, as roots can sense the presence of self and non-self roots, with the response being either stimulation of root growth or avoidance (de Kroon 2007, Semchenko et al. 2007b, Mommer et al. 2012). Finally, positive associations belowground can be due to root foraging ability, and an overlap in species micro-niches. Kesanakurti et al. (2011) found strong belowground segregation in a Canadian old field, although when they examined co-occurrences within families, they found that closely related species were more likely to coexist. They attributed this pattern to shared common traits and/or physiological tolerances. Four species pairs were negatively associated belowground (no association aboveground), which can be due to resource competition, allelopathy, or root behavioral ecology. In this case, perhaps opposing processes occurred aboveground (e.g., facilitation or micro-environmental filtering) that neutralized segregation that was observed belowground; indeed most of the negative associations included the Agrostis species group, which also had some positive associations aboveground. Negative associations may be species specific, and, in our case, many involved grasses and especially the Agrostis species group. Mommer et al. (2012) found that Agrostis stolonifera responded to nutrient rich patches through foraging ability, but when grown with a superior competitor, root growth and foraging activity occurred in less nutrient rich patches. Hence, root behavioral ecology to avoid neighbors may explain our negative associations for grasses found belowground.

Previous studies have found that spatial patterns in root and shoot communities are partly independent of each other (Pecháčková et al. 1999, Wildová 2004), and we show that assembly patterns (and the processes inferred from them) are not overlapping either. Moreover, we found evidence that biotic assembly processes aboveground became stronger when species were constrained by their belowground presence, providing good evidence that aboveground species coexistence may be limited by competition. Expanding on the concept of the belowground community as a type of species pool, we can consider aboveground species interactions as even more negative, than would be detected by examining aboveground data alone, because 10 species pairs that were positively associated belowground were randomly associated aboveground. Why is this pattern not reflected aboveground? It is likely that aboveground processes (e.g., competition for light) have neutralized this pattern. If this is so, then negative associations could be much more common aboveground than can be observed by aboveground sampling only, and may partially explain the lack of evidence for biotic assembly rules aboveground (Götzenberger et al. 2012).

Our study is the first to detail community assembly patterns of aboveground shoots and belowground roots and rhizomes in a natural community. We conclude that belowground assembly patterns differ from aboveground patterns; there is more evidence for abiotic and stochastic processes and less support for biotic processes. Our study details patterns in a small grassland, at one point in time, and it is currently unknown how general these findings are to other communities, and if patterns may change temporally. Moreover, differences may be found across environmental gradients (e.g., productivity) and with disturbance regimes. Further studies in more communities, including various gradients, and incorporating abundance measures (which are possible with these molecular techniques) will contribute greatly to our understanding of plant community assembly.

\section{ACKNOWLEDGMENTS}

This study was funded by the European Social Fund through a MOBILITAS postdoctoral grant (MJD47), by the European Union seventh framework project SCALES, FP7-226852; European Union sixth framework project ECOCHANGE, FP6-036866; the European Regional Development Fund (Center of Excellence FIBIR), and by the Estonian Science Foundation (grants 8323, 8613). We thank Mark Vellend for thoughtful discussion on this paper, and Triin Reitalu and two anonymous reviewers for useful comments on an earlier version of the manuscript.

\section{Literature Cited}

Bell, G. 2005. The co-distribution of species in relation to the neutral theory of community ecology. Ecology 86:1757-1770.

Bever, J. D. 2003. Soil community feedback and the coexistence of competitors: conceptual frameworks and empirical tests. New Phytologist 157:465-473.

Bever, J. D., I. A. Dickie, E. Facelli, J. M. Facelli, J. N. Klironomos, M. Moora, M. C. Rillig, W. D. Stock, M. Tibbett, and M. Zobel. 2010. Rooting theories of plant community ecology in microbial interactions. Trends in Ecology and Evolution 25:468-478.

Callaway, R. M. 1995. Positive interactions among plants. Botanical Review 61:306-349.

Carl, G., and I. Kühn. 2007. Analyzing spatial autocorrelation in species distributions using Gaussion and logit models. Ecological Modelling 207:159-170.

de Kroon, H. 2007. Ecology-How do roots interact? Science 318:1562-1563.

Farley, R. A., and A. H. Fitter. 1999. The responses of seven co-occurring woodland perennials to localised nutrient-rich patches. Journal of Ecology 87:849-859.

Frank, D. A., A. W. Pontes, E. M. Maine, J. Caruana, R. Raina, S. Raina, and J. Fridley. 2010. Grassland root communities: species distributions and how they are linked to aboveground abundance. Ecology 91:3201-3209.

Fransen, B., H. de Kroon, and F. Berendse. 2001. Soil nutrient heterogeneity alters competition between two perennial grass species. Ecology 82:2534-2546.

Gotelli, N. J. 2000. Null model analysis of species co-occurrence patterns. Ecology 81:2606-2621.

Gotelli, N. J., and D. J. McCabe. 2002. Species co-occurrence: a meta analysis of J. M. Diamond's assembly rule model. Ecology 83:2091-2096.

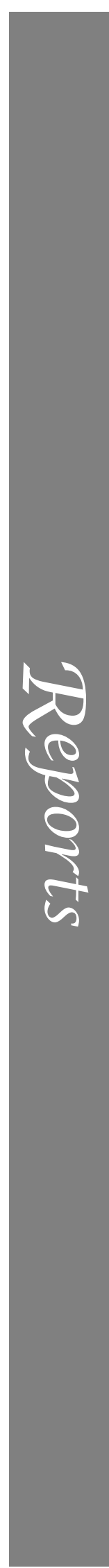


Götzenberger, L., et al. 2012. Ecological assembly rules in plant communities - approaches, patterns and prospects. Biological Reviews 87:111-127.

Grime, J. P. 1979. Plant strategies and vegetation processes. John Wiley, New York, New York, USA.

Grime, J. P. 2006. Trait convergence and trait divergence in herbaceous plant communities: mechanisms and consequences. Journal of Vegetation Science 17:255-260.

Hauggaard-Nielson, H., and E. S. Jensen. 2005. Facilitative root interactions in intercrops. Plant and Soil 274:237-250.

Hiiesalu, I., M. Öpik, M. Metsis, J. Davison, M. Vasar, M. Moora, M. Zobel, S. D. Wilson, and M. Pärtel. 2012. Plant species richness belowground: higher richness and new patterns revealed by next generation sequencing. Molecular Ecology. http://dx.doi.org/10.1111/j.1365-294x.2011.05390.x

Hodge, A. 2004. The plastic plant: root responses to heterogeneous supplies of nutrients. New Phytologist 162:9-24.

Keddy, P. A. 1992. Assembly and response rules: two goals for predictive community ecology. Journal of Vegetation Science 3:157-164

Kesanakurti, P. R., A. J. Fazekas, K. S. Burgess, D. M. Percy, S. G. Newmaster, S. W. Graham, S. C. H. Barrett, M. Hajibabaei, and B. C. Husband. 2011. Spatial patterns of plant diversity below-ground as revealed by DNA barcoding. Molecular Ecology 20:1289-1302.

Legendre, P., M. R. T. Dale, M.-J. Fortin, P. Casgrain, and J. Gurevitch. 2004. Effects of spatial structures on the results of field experiments. Ecology 85:3202-3214.

Maron, J. L., M. Marler, J. W. Klironomos, and C. C. Cleveland. 2011. Soil fungal pathogens and the relationship between plant diversity and productivity. Ecology Letters $14: 36-41$

Miklos, I., and J. Podani. 2004. Randomization of presenceabsence matrices: comments and new algorithms. Ecology 85:86-92.

Mommer, L. J., J. van Ruijven, H. de Caluwe, A. E. SmitTiekstra, C. A. M. Wagemaker, N. J. Ouborg, G. M. Bögemann, G. M. van der Weerden, F. Berendse, and H. de Kroon. 2010. Unveiling belowground species abundance in a biodiversity experiment: a test of vertical niche differentiation among grassland species. Journal of Ecology 98:1117-1127.

Mommer, L. J., J. van Ruijven, C. Jansen, H. M. van de Steeg, and H. de Kroon. 2012. Interactive effects of nutrient heterogeneity and competition: implications for root foraging theory. Functional Ecology 26:66-73.

Pärtel, M., R. Szava-Kovats, and M. Zobel. 2011. Dark diversity: shedding light on absent species. Trends in Ecology and Evolution 26:124-128.

Pecháčková, S., H. J. During, V. Rydlová, and T. Herben. 1999. Species-specific spatial pattern of below-ground plant parts in a montane grassland community. Journal of Ecology 87:569-582.

Petermann, J. S., A. J. F. Fergus, C. Roscher, L. A. Turnbull, A. Weigelt, and B. Schmid. 2010. Biology, chance, or history? The predictable reassembly of temperate grassland communities. Ecology 91:408-421.
R Development Core Team. 2009. R: A language and environment for statistical computing. R Foundation for Statistical Computing, Vienna, Austria. www.r-project.org

Reynolds, H. L., A. Packer, J. D. Bever, and K. Clay. 2003. Grassroots ecology: plant-microbe-soil interactions as drivers of plant community structure and dynamics. Ecology 84:2281-2291.

Schamp, B. S., and L. W. Aarssen. 2009. The assembly of forest communities according to maximum species height along resource and disturbance gradients. Oikos 118:564-572.

Schenk, H. J. 2006. Root competition: beyond resource depletion. Journal of Ecology 94:725-739.

Schnitzer, S. A., J. N. Klironomos, J. HilleRisLambers, L. L. Kinkel, P. B. Reich, K. Xiao, M. C. Rillig, B. A. Sikes, R. M. Callaway, S. A. Mangan, E. H. van Nes, and M. Scheffer. 2011. Soil microbes drive the classic plant diversityproductivity pattern. Ecology 92:296-303.

Seabloom, E. W., O. Bjørnstad, N. B. B. Bolker, and O. J. Reichman. 2005. Spatial signature of environmental heterogeneity, dispersal, and competition in successional grasslands. Ecological Monographs 75:199-214.

Semchenko, M., M. J. Hutchings, and E. A. John. $2007 a$. Challenging the tragedy of the commons in root competition: confounding effects of neighbour presence and substrate volume. Journal of Ecology 95:252-260.

Semchenko, M., E. A. John, and M. J. Hutchings. $2007 b$. Effects of physical connection and genetic identity of neighbouring ramets on root-placement patterns in two clonal species. New Phytologist 176:644-654.

Tilman, D. 1982. Resource competition and community structure. Princeton University Press, Princeton, New Jersey, USA.

Weiner, J. 1990. Asymmetric competition in plant populations. Trends in Ecology and Evolution 5:360-364.

Wildová, R. 2004. Below-ground spatial pattern of rhizomes in a grassland community and its relevance to above-ground spatial pattern. Plant Ecology 174:321-338.

Wildová, R., I. Wild, and T. Herben. 2007. Fine-scale dynamics of rhizomes in a grassland community. Ecography 30:264276.

Wilson, J. B. 1989. A null model of guild proportionality, applied to stratification of a New Zealand temperate rain forest. Oecologia 80:263-267.

Wilson, J. B., H. Gitay, and A. D. Q. Agnew. 1987. Does niche limitation exist? Functional Ecology 1:391-387.

Wilson, J. B., and M. T. Sykes. 1988. Some tests for niche limitation by examination of species diversity in the Dunedin area, New Zealand. New Zealand Journal of Botany 26:237244.

Wilson, J. B., and R. J. Whittaker. 1995. Assembly rules demonstrated in a saltmarsh community. Journal of Ecology 83:801-807.

Zobel, K., and M. Zobel. 1988. A new null hypothesis for measuring the degree of plant community organisation. Vegetatio 75:17-25.

\section{Supplemental Material}

\section{Appendix A}

Molecular analysis (Ecological Archives E093-113-A1).

\section{Appendix B}

Significant pairwise species associations and $P$ values from spatially informed generalized estimation equations (GEEs) for above- and belowground data (Ecological Archives E093-113-A2). 